\title{
Prevalencia de caries de infancia temprana severa y factores de riesgo asociados en un grupo de niños del área metropolitana de Guatemala
}

\author{
Bárbara Bustamante Castillo, ${ }^{1}$ \\ Ernesto Villagrán Colón, ${ }^{2}$ \\ Alfredo Moreno Quiñónez, ${ }^{3}$ \\ Michelle Bustamante-Castillo. ${ }^{4}$
}

\section{Resumen}

Objetivo: Determinar la prevalencia y severidad de caries de infancia temprana severa y factores de riesgo asociados en una poblaciónn de niños de 6-36 meses de edad, que asisten a guarderías estatales del área metropolitana de la ciudad de Guatemala. Materiales y métodos: Diseño analítico transversal. Se examinaron clínicamente 110 niños de 13 a 36 meses de edad para determinar el estado de caries según los criterios del Sistema Internacional de Detección y Valoración de Caries (Kappa 0.69). Además, se determinaron las características sociodemográficas, hábitos de alimentación e higiene dental a través de una entrevista a las madres de los participantes y se realizó análisis descriptivo de estas variables. Para determinar la relación entre variables se aplicaron las pruebas estadísticas U de Man Whitney y Tau-b de Kendall. Resultados: Se encontró una prevalencia de caries de infancia temprana severa de $81.8 \%$. En promedio cada individuo presentó 6.74 lesiones cariosas (IC 95\% 5.62 - 7.92). El número promedio de lesiones no cavitadas fue 6.06 y de lesiones cavitadas fue 2.51. Se encontró que la edad y el porcentaje de superficies dentarias con presencia de placa están significativamente asociados con las caries de infancia temprana severa. Conclusiones: La alta prevalencia de caries $(81.8 \%)$ y el carácter reversible de $2 / 3$ de las lesiones encontradas demanda intervenciones preventivas de salud dental en esta población.

Palabras clave: Caries dental, infancia temprana, factores de riesgo, prevención primaria.

\footnotetext{
${ }^{1}$ Odontóloga. Colonia Roosevelt, Ciudad de Guatemala, Guatemala.

${ }^{2}$ MSc. Facultad de Odontología Universidad San Carlos, Ciudad de Guatemala, Guatemala.

${ }^{3}$ MSc. Phd. Facultad de Ciencias Médicas, Escuela de estudios de Post grado, Universidad San Carlos, Ciudad de Guatemala, Guatemala.

${ }^{4}$ Instituto de Investigaciones, Centro Universitario de Zacapa, Universidad San Carlos, Ciudad de Guatemala, Guatemala.
} 
Artigo original

\section{Prevalência de cárie precoce na infância e fatores de risco associados em um grupo de crianças na região metropolitana da Guatemala}

Resumo

Objetivo: Determinar a prevalência e a gravidade de cáries precoce na infância e fatores de risco associados em uma população de crianças de 6 a 36 meses que frequentam creches estaduais na região metropolitana da Cidade da Guatemala. Material e métodos: Projeto analítico transversal 110 crianças de 13 a 36 meses de idade foram examinadas clinicamente para determinar o status de cárie de acordo com os critérios do Sistema Internacional de Detecção e Avaliação de Cárie (Kappa 0,69). Além disso, as características sociodemográficas, hábitos alimentares e higiene dental foram determinadas por meio de entrevista com as mães dos participantes e análise descritiva dessas variáveis. Para determinar a relação entre as variáveis, foram aplicados os testes estatísticos U de Man Whitney e Tau-b de Kendall. Resultados: A prevalência de cárie na primeira infância foi de $81,8 \%$. Em média, cada indivíduo apresentou 6,74 lesões de cárie (IC95 \% 5,62 - 7,92). $\mathrm{O}$ número médio de lesões não cavitadas foi de 6,06 e as cavitadas foram de 2,51. Verificou-se que a idade e a porcentagem de superfícies dentárias com presença de placa estão significativamente associadas à cárie precoce. Conclusões: A alta prevalência de cárie encontrada $(81,8 \%)$ e do tipo reversível foi de $2 / 3$ das lesões encontradas demandam intervenções preventivas de saúde bucal nessa população.

Palavras chaves: Cárie dentária na primeira infância, fatores de risco, prevenção primária.

Original article

\section{Prevalence of severe early childhood caries and associated risk factors in a group of children in the metropolitan area of Guatemala}

\begin{abstract}
Objective: To determine the prevalence and severity of early childhood caries and the associated risk factors, in a population of 6 to 36 months old children that attend governmental daycares in the metropolitan area of Guatemala City. Methods: Cross-sectional analytical
\end{abstract}

design 110 children from 13 to 36 months of age were clinically examined to determine caries status according to the criteria of the International Caries Detection and Assessment System (Kappa 0.69). In addition, sociodemographic characteristics, eating and dental hygiene habits were established through a survey done to the mothers of the participants. 
To determine the relationship between variables, the statistical tests $U$ of Man Whitney and Tau-b of Kendall were applied. Results: There was an $81.8 \%$ of prevalence found of severe early childhood caries. In average, each subject showed 6.74 carious lesions (IC 95\% 5.62 - 7.92). The average number of noncavitated lesions was 6.06 and 2.51 for cavitated lesions. It was found that age $(\tau \mathrm{b}=.224, \mathrm{p}=.001)$ and the percentage

\section{Introducción}

La Caries de Infancia Temprana Severa (CIT-S) constituye cualquier signo de caries de superficie lisa, con o sin cavidad en niños menores de 36 meses de edad. ${ }^{1}$ Se presenta como una patología no auto limitante que destruye los tejidos dentarios. La primera consecuencia asociada, el dolor, afecta la ingesta de alimentos y altera los patrones de sueño y juego. ${ }^{2}$ Se le asocia a la malnutrición y se sabe que la inflamación crónica por pulpitis y abscesos dentales suprime el crecimiento y reduce la hemoglobina como resultado de una producción de eritrocitos deprimida que pueden contribuir al bajo peso y/o talla en niños. ${ }^{3}$ Con frecuencia la CIT-S puede conducir a la pérdida de los dientes frontales afectando el desarrollo del habla. Las repercusiones psicosociales de la CIT-S severa incluyen baja autoestima, renuencia a hablar o sonreír en público, recibir burlas de otros y estrés relacionado a la necesidad de tratamiento dental como extracciones. ${ }^{4}$ Entre los efectos a largo plazo de esta patología se encuentran las relacionadas con la malnutrición, mayor riesgo de desarrollo de caries en dientes permanentes y mal posición dentaria. ${ }^{5}$ of dental surface with plaque $(\tau b=0.352$ $\mathrm{p}=.002)$ are significantly associated with severe early childhood caries. Conclusion: The high prevalence from found caries $(81.8 \%)$ and the reversible type of $2 / 3$ from the found lesions, show a need of preventive interventions of dental health in this population.

Key words: Dental caries, early childhood, risk factors, primary prevention.

La CIT-S severa tiene una etiología compleja con influencias biológicas, conductuales y sociodemográficas. La evidencia sugiere que los niños son más propensos a desarrollar caries si adquieren Streptococcus mutans a una edad temprana, aunque esto es influenciado por otros factores, como la higiene bucal, exposición a fluoruros tópicos, la dieta, el acceso a cuidados de salud oral, el estatus socioeconómico, la etnicidad y la alfabetización en salud. Existe evidencia que la CIT-S afecta de manera desproporcional a individuos que viven en condiciones económicas desfavorables o que pertenecen a grupos étnicos marginados. ${ }^{5,6}$

La prevalencia de este tipo de lesiones dentales varia ampliamente entre poblaciones. En América se estima una prevalencia conjunta aproximada de $70 \%{ }^{7}$ Países como Brasil reportan una prevalencia de hasta $52 \%$; mientras que en México y los países Centroamericanos es aún más alta (entre $60 \%$ y $93 \%$ ). ${ }^{8}$ En Guatemala, un estudio de riesgo de caries realizado en una población de 218 niños menores de 3 años, encontró una prevalencia de $53 \%$ de lesiones de desmineralización congruentes con caries. ${ }^{9}$ 
Aunque en la mayoría de los casos la CIT-S es prevenible o tratable, cerca del $90 \%$ de las lesiones no reciben tratamiento. ${ }^{10}$ En Guatemala el Ministerio de Salud Pública y Asistencia Social -MSPAS- no cuenta con programas de prevención de caries en menores de 7 años, ni se dispone de datos necesarios para planificar estrategias de atención sanitaria. Por lo anterior, se realizó un estudio transversal con el objetivo de estimar la prevalencia y severidad de la CIT-S y evaluar las posibles correlaciones con factores socioeconómicos y de comportamiento, específicamente en niños de 6-36 meses que asisten a los Centros de Atención de la Secretaria de Bienestar Social de la Presidencia del área Metropolitana del país. La información recabada en este estudio puede ser empleada en el diseño de actividades de promoción y prevención de caries en beneficio de niños menores de tres años del país.

\section{Materiales y métodos}

\section{Población y sitio de estudio}

El estudio se llevó a cabo en Centros de Atención Integral -CAI- de la Secretaria de Bienestar Social de la Presidencia -SBS- de Guatemala, ubicados en el área metropolitana del país. La Secretaria de Bienestar Social de la presidencia es un órgano administrativo gubernamental dependiente del Organismo Ejecutivo que tiene a su cargo 15 Centros de Atención Integral -CAI- en la Ciudad de Guatemala. En ellos, se ofrece cuidado diurno diario, alimentación y educación gratuita a niños de entre 8 meses a 12 años de edad, hijos de padres y madres trabajadoras mientras ellos desempeñan su jornada laboral.

Los datos para este estudio se recopilaron entre septiembre y octubre del año 2017.
La población de interés estuvo formada por 228 sujetos de entre 13 y 36 meses de edad con uno o más dientes primarios presentes en boca e inscritos en algúnn CAI del área metropolitana de la ciudad de Guatemala. La muestra estudiada a partir de esta población estuvo formada por 110 niños de 13 a 36 meses de edad, divididos en 2 grupos de edades: 1324 meses de edad $(34.5 \% ; 38)$ y de $25-36$ meses de edad $(65.5 \% ; 72)$. Se examinaron a 55 niñas (50\%) y 55 niños (50\%).

El tamaño de la muestra representativa de este estudio se obtuvo de manera aleatoria empleando un nivel de confianza del $90 \%$, un margen de error muestral de $0.07 \%$ y asumiendo una prevalencia de lesiones de caries del 50\%. Previendo pérdida de sujetos en la muestra, el tamaño de la muestra se incrementó en un $15 \%$, resultando en un total de 110 niños.

Este estudio fue aprobado por el Comité de Bioética en Investigación en Salud de la Facultad de Ciencias Médicas de la Universidad de San Carlos de Guatemala. Previo a los exámenes clínicos y aplicación de cuestionarios se obtuvieron consentimientos informados de los padres.

\section{Examen clínico}

Con el fin de garantizar juicios clínicos consistentes una examinadora principal y un experto en ICDAS II evaluaron 24 niños de características similares a aquellos incluidos en la muestra, alcanzando un grado de concordancia general de 77\% y un valor de test Kappa de 69\%.

Tanto el examen clínico de adultos como el de menores se llevó a cabo en las instalaciones de los CAI. Se utilizó un espejo intraoral No. 5, sonda periodontal 
No. 11 OMS y lámpara intraoral de luz blanca. Para cada individuo se utilizó un set de instrumentos estériles. No se tomaron radiografías. Entre cada examen se desinfectó la lámpara intraoral y superficies de trabajo. La investigadora examinó a cada paciente y narró los hallazgos a una asistente quien registró la información en formatos impresos especialmente diseñados para este estudio.

Durante el examen clínico a madres de los menores, se registró la presencia, de por lo menos, una caries activa en la corona dental según los criterios ICDAS II (Tabla 1).

El examen clínico en los niños en estudio se realizó utilizando la técnica de rodilla a rodilla. Inició con el registro de dientes y superficies por dientes presentes, luego, se aplicó líquido revelador de placa dentobacteriana con un pincel sobre todas las superficies dentales y se registraron las superficies bucales y linguales que se tiñeron. A continuación, la investigadora y una asistente cepillaron los dientes de los menores con cepillo y pasta dental para remover el colorante y placa dentobacteriana, entonces se procedió al registro de superficies afectadas por caries y la gravedad de las lesiones según criterios del Sistema Internacional de Detección y Diagnóstico de Caries II -ICDAS II- (Tabla 2).

Debido a limitaciones logísticas, las superficies evaluadas fueron secadas con gasa estéril y no con un chorro de aire de 5 segundos de duración como lo recomiendan los criterios ICDAS II.

\section{Entrevista a madres de familia}

Con el fin de obtener información sobre los factores socioeconómicos y conductuales
Tabla 1. Criterios de actividad de la caries coronal según ICDAS II.

\begin{tabular}{|c|c|c|}
\hline \multirow{2}{*}{$\begin{array}{l}\text { Código } \\
\text { ICDAS }\end{array}$} & \multicolumn{2}{|c|}{ Características de la lesión. } \\
\hline & Lesión activa & Lesión inactiva \\
\hline 203 & $\begin{array}{l}\text { La superficie } \\
\text { del esmalte es } \\
\text { blanquecina / } \\
\text { amarillenta opaca } \\
\text { con pérdida de } \\
\text { brillo; se siente } \\
\text { irregular al } \\
\text { deslizar la punta } \\
\text { de la sonda sobre } \\
\text { la superficie. } \\
\text { La lesión se } \\
\text { encuentra en } \\
\text { un área de } \\
\text { estancamiento de } \\
\text { la placa, es decir: } \\
\text { fosas y fisuras, } \\
\text { cerca de la zona } \\
\text { gingival y } \\
\text { Superficie } \\
\text { aproximada por } \\
\text { debajo del punto } \\
\text { de contacto. }\end{array}$ & $\begin{array}{l}\text { La superficie } \\
\text { del esmalte es } \\
\text { blanquecina, } \\
\text { parda o negra. } \\
\text { El esmalte puede } \\
\text { ser brillante y se } \\
\text { siente duro y liso } \\
\text { cuando la sonda } \\
\text { se desliza sobre la } \\
\text { superficie. } \\
\text { En superficies } \\
\text { lisas, la lesión } \\
\text { de caries se } \\
\text { encuentra } \\
\text { típicamente } \\
\text { lejos del margen } \\
\text { gingival. }\end{array}$ \\
\hline 4 & $\begin{array}{l}\text { Probablemente } \\
\text { activa. }\end{array}$ & \\
\hline 506 & $\begin{array}{l}\text { La cavidad se } \\
\text { siente suave } \\
\text { o correosa } \\
\text { al sondear } \\
\text { suavemente la } \\
\text { dentina. }\end{array}$ & $\begin{array}{l}\text { La cavidad puede } \\
\text { ser brillante y } \\
\text { se siente dura } \\
\text { al sondear } \\
\text { suavemente } \\
\text { dentina. }\end{array}$ \\
\hline
\end{tabular}

*Fuente: https://www.icdas.org/*

relacionados con la presencia y gravedad de CIT-S en los niños participantes en el estudio, se aplicó un cuestionario en forma de entrevista a sus madres. El cuestionario recolectó datos sobre la edad en meses de los menores al momento de la evaluación, sexo y nivel educacional de la madre categorizado como: ninguno, preprimaria, primaria, básicos, diversificado, universitario $u$ otros. Se indagó sobre el acceso a la Canasta Básica Vital -CBV. La 
Tabla 2. Códigos ICDAS II y su descripción.

\begin{tabular}{|l|l|}
\hline Código & Descripción \\
\hline 0 & $\begin{array}{l}\text { Superficie dental sin evidencia de } \\
\text { lesión de caries dental después del } \\
\text { secado con gasa* }\end{array}$ \\
\hline 1 & $\begin{array}{l}\text { Mancha blanca / marrón en esmalte } \\
\text { seco }\end{array}$ \\
\hline 2 & $\begin{array}{l}\text { Cambio de coloración, lesión de caries } \\
\text { dental blanca o marrón, perceptible } \\
\text { visualmente en esmalte sin necesidad } \\
\text { de secar la superficie (húmeda), } \\
\text { tanto en superficies lisas como } \\
\text { proyectándose en la entrada de las } \\
\text { fosas y fisuras }\end{array}$ \\
\hline 3 & $\begin{array}{l}\text { Ruptura localizada del esmalte } \\
\text { por lesión de caries dental, sin } \\
\text { dentina visible, discontinuidad en la } \\
\text { superficie del esmalte. }\end{array}$ \\
\hline $\begin{array}{l}\text { Sombra oscura subyacente desde la } \\
\text { dentina con o sin ruptura localizada } \\
\text { del esmalte. }\end{array}$ \\
\hline $\begin{array}{l}\text { Lesión de caries dental cavitada con } \\
\text { dentina expuesta en la base de la } \\
\text { cavidad, comprometiendo menos de } \\
\text { la mitad de la superficie. }\end{array}$ \\
\hline mitad o más de la superficie \\
\hline 5
\end{tabular}

*Fuente: https://www.icdas.org/*

CBV es el conjunto de bienes y servicios que satisfacen las necesidades de alimentación, vestuario, vivienda, salud, educación etc. de los integrantes de un grupo familiar. Según estimaciones del Instituto Nacional de Estadística -INE- de Guatemala durante el trimestre anterior a la entrevista la CVB tenía un costo mensual aproximado de Q. 7,483.00. Para fines de esta investigación el acceso a la CVB se clasificó según ingresos familiares mensuales combinados menores, iguales o mayores a Q. 7, 500.00.

También se registró la frecuencia de ingesta de alimentos cariogénicos para lo cual se definió como cariogénico cualquier alimento que contiene sacarosa. Se consideró la frecuencia de ingesta cariogénica dentro y fuera del CAI, incluyendo el menú de alimentación infantil estandarizado que rige el contenido de desayuno, almuerzo y dos refacciones que reciben los niños que asisten a la institución. Se pidió a las madres que proporcionaran información sobre la frecuencia de ingesta de biberón nocturno con líquidos azucarados $\mathrm{y}$ lactancia materna nocturna. Por último, las madres y niñeras del CAI reportaron la frecuencia con que cepillaron con pasta dental fluorada a cada menor.

\section{Análisis de datos}

Se determinaron frecuencias y porcentajes para todas las variables estudiadas.

La presencia de placa dentobacteriana sobre las superficies dentales se evaluó con el Índice de Placa de O'Leary modificado. Para su construcción se determinó el número superficies bucales y linguales presentes en cada niño y el porcentaje de esas superficies teñidas con liquido revelador de placa dentobacteriana. Se excluyeron las superficies interproximales para reducir el tiempo de examen, favorecer la cooperación y disminuir el estrés asociado de los menores. Se consideró como aceptable entre 0.0\% $-12.0 \%$ de las superficies dentales cubiertas con placa, cuestionable entre $13.0 \%-23.0 \%$ y deficiente de $24.0 \%-100.0 \%$ de las superficies cubiertas con placa dentobacteriana. ${ }^{11}$ 
El índice ceo-d ${ }^{12}$ se construyó con los datos recolectados según criterios ICDAS II. Para esto se establecieron equivalencias entre el índice ceo-d e ICDAS II Los códigos ICDAS II (0-2) se agruparon en el código ceo-d "A "o corona sana. Los códigos ICDAS II (36) se agruparon en el código ceo-d " $\mathrm{C}$ " o corona cariada. ${ }^{13}$ Dado que en este estudio no se detectaron dientes perdidos por caries u obturadas, la prevalencia de caries según ICDAS II resulta de la sumatoria de los individuos con por lo menos una superficie dental con caries (códigos ICDAS II 1-6) expresada como porcentaje del total de individuos en el estudio. Mientras que la prevalencia de caries en la población, según el índice ceo-d, resulta de la sumatoria de los individuos con por lo menos una superficie dental con caries (sumatoria de "C" códigos ICDAS II 3-6) expresada como porcentaje del total de individuos en el estudio. Únicamente se consideraron caries activas (Tabla 2). Esta métrica fue basada en muestreo aleatorio con reemplazo o "bootstrapping".

La severidad de las lesiones por caries fue determinada según ICDAS II que categoriza con códigos 1 a 6 las lesiones de caries según el grado de avance, desde estadios tempranos de desmineralizaciónn (código 1-2) hasta lesiones cavitadas con dentina visible y que comprometen la mitad o más de la superficie dental (código 6, Tabla 2). Esta métrica fue basada en muestreo aleatorio con reemplazo o "bootstrapping".

Para resaltar el carácter reversible o irreversible de la lesión de caries, las lesiones cavitadas y no cavitadas fueron registradas por separado bajo la siguiente codificación: d (0) refleja dientes sanas, d (2) refleja los códigos ICDAS 1-2 (lesiones no cavitadas) y d (3-6) indica los códigos ICDAS 3-6 (lesiones cavitadas).

Para determinar la asociación entre la frecuencia de caries según ICDAS II y características socioeconómicas y conductuales de la población se calculó el coeficiente de correlación tau-b $(\tau b)$ de Kendall. Se mantuvo el nivel de medición ordinal de las variables: acceso a la canasta básica vital -CVB- (categorizado como ingresos familiares mensuales combinados menores o iguales o mayores a Q.7, 500.00), nivel educacional de la madre (categorizado como menos de 12 años de educación y 12 o más años de educación), ingesta de dieta cariogénica durante el día (categorizado como 3-5 veces por día y 5 o más veces por día), consumo de biberón nocturno con azúcar (categorizado como 1-3 veces por noche $y>3$ veces por noche), frecuencia de cepillado dental realizado por un adulto con pasta dental fluorada (categorizado como $0-2$ veces por día y 3 ó más veces por día),niveles de placa dentobacteriana (categorizado aceptable $<12 \%$ de superficies con placa dentobacteriana y deficiente $\geq 12 \%$ de superficies con placa dentobacteriana) y consumo de lecha materna nocturna (categorizada como 1 vez por noche, más de 1 vez por noche). La variable edad fue categorizada como 13-24 meses de edad y 25-36 meses de edad. La variable frecuencia de caries fue categorizada como: igual o menor de 5 superficies con caries y más de 5 superficies con caries según criterios ICDAS II. Para el cálculo de los datos se contravino la suposición de distribuciones monótonas.

Con el fin de comparar las diferencias entre grupos se utilizó la prueba U de Mann-Whitney para lo cual las variables 
dependientes fueron dicotomizadas. La variable acceso a la CBV se dicotomizó como: sin acceso a la CVB (ingresos familiares menores a Q.7,500.00) y con acceso a la CVB (ingresos familiares iguales o mayores Q.7,500.00). El nivel educacional de la madre se dicotomizó según los años de escolaridad alcanzada con un punto de corte de 12 años: menos de 12 años de escolaridad y 12 o más años de escolaridad.

La ingesta de pacha o biberón nocturno con líquidos azucarados se dividió en 2 grupos: consume y no consume. La variable frecuencia de ingesta de dieta cariogénica durante el día se dividió en 2 grupos: 0-3 veces al día y 4 o más veces al día. La frecuencia de ingesta de lactancia materna durante la noche fue dividida en 2 categorías: consume o no consume. La frecuencia de cepillado dental con pasta con flúor realizado por un adulto durante el día fue dividida en: 0-1 vez por día y 2 o más veces por día. La presencia de placa dentobacteriana en superficies dentales según el índice de O'Leary modificado fue agrupada en aceptable $<12 \%$ de superficies con placa dentobacteriana o deficiente $\geq 12 \%$ de superficies con placa dentobacteriana. La presencia de caries dental activa en la madre se dividió en 2 grupos: presenta o no presenta.

Para ambas pruebas el valor $\mathrm{p}<0.05$ fue considerado estadísticamente significativo. El procesamiento y análisis de la información se realizó con el programa para análisis Epi Info versión 7.2 .

\section{Resultados}

Según ICDAS II la prevalencia de caries en la población infantil evaluada fue $81.8 \%$ (105). Un mayor porcentaje de niños $(85.5 \%$; 47) presentaron caries en comparación con las niñas $(78.2 \% ; 43)$. En ambos sexos, la prevalencia de caries fue mayor en el grupo de 25-36 meses de edad (niños 94.2\%; 33 y niñas $81 \%$; 30) comparado con el grupo de 13-24 meses de edad (niños 70\%; 14 y niñas $72.2 \%$; 13). La prevalencia según ceo-d fue de $50 \%$ (55). ICDAS II también registró un mayor promedio de dientes afectados por niño en comparación con ceo-d: 6.74 y 1.94 respectivamente.

Según ICDAS II, en promedio cada individuo presentó 6.74 lesiones cariosas (IC 95\% 5.62 - 7.92). La frecuencia de caries más elevada se encontró en el grupo de 25-36 meses de edad en ambos sexos: niños 8.23 (IC 95\% 6.54 - 10.12) y niñas 7.32 (5.67 - 8.98).

Respecto a la severidad de la caries, según ICDAS II el número promedio lesiones no cavitadas fue 6.06 (código 12) y lesiones cavitadas fue 2.59 (códigos 3-6). Este último correspondería al promedio de lesiones según el índice ceo. El mayor número de superficies cavitadas se encontró en niñas de 25-36 meses de edad (3.73).

En cuanto a las características socioeconómicas de la población evaluada, el 44.5\% (49) de las madres entrevistadas refirió haber alcanzado doce o más años de educación (diversificado o universitario) y un $90 \%$ (99) tener ingresos mensuales familiares 
combinados por debajo del costo de la Canasta Básica Vital -CBV- (Q.7,500 mensuales).

Durante la entrevista se encontró que el 59.1\% (65) de los menores consumen alimentos o bebidas azucaradas más de 5 veces al día, con una media de 6.32 veces por día. De todos los evaluados el $25.4 \%$ (28) consume biberón nocturno con líquidos azucarados y el $96.4 \%$ (27) de este grupo con una frecuencia de entre 1-3 veces por noche. El $17.3 \%$ (19) de los niños es alimentado con lactancia materna durante la noche y de estos el 57.9\% (1) una vez por noche.

En cuanto a hábitos de higiene, el 26.4\% (29) de los miembros de la población son cepillados ninguna o una vez al día, el restante $73.6 \%$ (81) es cepillado 2 o más veces por día. Durante la evaluación oral clínica se evidenció que el $89.1 \%$ de la población presentó niveles deficientes de placa dentobacteriana, es decir, entre $24.0 \%-100.0 \%$ de las superficies bucales y linguales cubiertas de placa dentobacteriana según el Índice de placa de O’Leary modificado.

Al examen clínico el 65\% (72) de las madres presentó alguna caries activa según los criterios ICDAS II.

Se encontró asociación estadísticamente significativa entre la frecuencia de caries en la población y la edad $(\tau b=0.224$, $\mathrm{p}=.001)$ y entre la frecuencia de caries en la población y el porcentaje de superficies dentarias con presencia de placa dentobacteriana ( $\tau b=0.352 \mathrm{p}=0.002)$. No se encontró relación significativa de ninguna otra variable sobre la frecuencia de caries de la población (Tabla 3).
Tabla 3. Factores de riesgo asociados a la frecuencia de caries.

\begin{tabular}{|c|c|c|c|}
\hline \multirow{2}{*}{ Variable } & \multicolumn{2}{|c|}{ Prueba estadística } & \multirow{2}{*}{$\mathrm{p}$} \\
\hline & $\tau b$ & $\mathrm{U}$ & \\
\hline Edad & 0,224 & & $0,001^{*}$ \\
\hline Sexo & & $1.625,05$ & 0,497 \\
\hline $\begin{array}{l}\text { Acceso a la } \\
\text { Canasta Básica } \\
\text { Vital -CBV- } \\
\end{array}$ & $-0,013$ & & 0,873 \\
\hline $\begin{array}{l}\text { Años de } \\
\text { escolaridad } \\
\text { alcanzados por la } \\
\text { madre }\end{array}$ & $-0,137$ & & 0,069 \\
\hline $\begin{array}{l}\text { Ingesta de dieta } \\
\text { cariogénica } \\
\text { durante el día } \\
\end{array}$ & 0,011 & & 0,872 \\
\hline $\begin{array}{l}\text { Consumo de } \\
\text { biberón nocturno } \\
\text { con azúcar }\end{array}$ & 0,044 & & 0,689 \\
\hline $\begin{array}{l}\text { Frecuencia de } \\
\text { cepillado dental }\end{array}$ & 0,160 & & 0,027 \\
\hline $\begin{array}{l}\text { Presencia de caries } \\
\text { activa en la madre }\end{array}$ & & $1,193.00$ & 0,500 \\
\hline $\begin{array}{l}\text { Niveles de placa } \\
\text { dentobacteriana }\end{array}$ & 0,352 & & $0,002^{*}$ \\
\hline $\begin{array}{l}\text { Consumo de leche } \\
\text { materna nocturna }\end{array}$ & $-0,118$ & & 0,433 \\
\hline
\end{tabular}

Fuente: construcción propia con datos del estudio.

\section{Discusión}

En este estudio el ICDAS II registró mayor prevalencia de caries y mayor número de dientes afectados por caries por niño en comparación con ceo-d. Esto debido a que el ceo-d registra únicamente lesiones que han roto la integridad del esmalte, mientras que ICDAS II adicionalmente registro los estadios tempranos de desarrollo de la enfermedad restringidos a procesos tempranos de desmineralización. ${ }^{14}$ Por otro lado, un número relativamente 
bajo de niños presentó lesiones con involucramiento de dentina. En el estudio se encontró un mayor número de lesiones no cavitadas que cavitadas, hallazgo similar a lo reportado por otros autores. ${ }^{15}$ En este estudio, ICDAS II permitió determinar la carga real de caries, establecer de manera diferencial la necesidad de intervenciones preventivas y curativas y posibilitó medir el éxito de dichas intervenciones en términos de incidencia y progresión de las lesiones. El índice ceo-d subestimó la carga real de la enfermedad en esta población, compuesta en su mayoría por lesiones restringidas a esmalte en estadios tempranos de desmineralización.

Se encontró una asociación entre la edad y la frecuencia de caries. Esto era de esperarse ya que la edad es un predictor de caries dental en dentición primaria. ${ }^{16}$ Usualmente, los niños con una experiencia temprana de caries tienen un alto riesgo de progresión de la enfermedad. ${ }^{16}$ El hallazgo de que la frecuencia y gravedad de las lesiones cariosas aumenta con la edad es congruente con la naturaleza acumulativa y crónica de la caries dado que tanto ICDAS II como ceo-d miden la experiencia de caries pasadas y presentes. ${ }^{17}$ Sin embargo, el hecho de que sean índices acumulativos no significa que la experiencia de caries no pueda mantenerse estable a lo largo del tiempo, lo que indica que no se han desarrollado más caries o progresado las existentes. ${ }^{18}$

Estos hallazgos contrastan con estudios que no han encontrado un aumento progresivo del número de dientes cariados en niños al incrementar su edad. ${ }^{19}$
Al igual que en otros estudios no se encontró diferencias en la frecuencia de caries entre sexos en la población estudiada. ${ }^{20}$ La erupción dentaria suele ocurrir a una edad más temprana en mujeres que en hombres, por lo que los dientes de estas están expuestos al ambiente bucal, a las bacterias y a los sustratos bacterianos durante más tiempo que los dientes de un hombre de la misma edad. ${ }^{21}$ Un estudio en niños de 12 años en Tiquisate, Guatemala, ${ }^{22}$ sugiere que las diferencias en la experiencia de caries entre sexos podrían asociarse mayormente a factores genéticos como el gen Amelogenin (AMELX) que reside en el brazo $p$ del cromosoma $X$ y que contribuye a la formación del esmalte en la dentición. Una mutación / deleción en el gen AMELX resulta en amelogénesis imperfecta ligada a X y una mayor susceptibilidad a la caries.

Los factores socioeconómicos, acceso a la Canasta Básica Vital -CBV- y nivel educacional de la madre tampoco mostraron asociación con la frecuencia de caries de la población evaluada. En general, la literatura reporta asociaciones inversas significativas entre los niveles de educación de los padres, los ingresos del hogar y la prevalencia de caries dental pues influyen en la ingesta de azúcar y hábitos de higiene oral. ${ }^{23} \mathrm{La}$ incongruencia del presente estudio y estos hallazgos puede estar relacionada con el hecho de que la mayoría de los niños evaluados no contaban con acceso a la Canasta Vital Básica lo que pudo dificultar medir la diferencia de efecto sobre la frecuencia de caries entre grupos. El hecho que todos los individuos examinados reciban una dieta estandarizada, 5 de 7 días a la semana, pudo haber reducido el efecto mediador de la educación de los padres sobre la dieta de los hijos. 
En este estudio no se encontró relación entre la frecuencia de consumo de azúcar y la frecuencia de caries de la población estudiada. Estos resultados coinciden con los reportados en otros estudios en Latinoamérica. $^{24}$

La evidencia más reciente relaciona tanto la ingesta total de azúcar en gramos como porcentaje energético total y la frecuencia de ingesta de azúcar como factores que pueden influir en la cariogenicidad de la placa. ${ }^{25}$

El consumo de leche materna justo antes de dormir o durante la noche y tras la cual no ocurre cepillado dental tampoco se relacionó con la frecuencia de caries de la población estudiada. La evidencia de asociación entre estos dos factores sugiere un efecto protector hasta los 12 meses para luego convertirse en un factor de riesgo. ${ }^{26}$ Otras investigaciones ${ }^{26-27}$ identificaron tres factores relacionados con la lactancia materna y/o la alimentación con biberón como factores de riesgo para la caries dental: duración de la lactancia mayor de 18 meses, utilizada para alimentar o dejar de llorar durante la noche y para dormir al niño infante. Por ejemplo, un estudio encontró que entre los niños amamantados menos de 12 meses, aquellos alimentados por la noche o más con frecuencia tuvieron un mayor riesgo de caries, sin embargo, el aumento del riesgo podría deberse a factores de confusión no medidos, incluida la dieta de azúcares y prácticas de higiene oral. ${ }^{28}$ En este sentido, la muestra evaluada en el presente estudio no contó con individuos menores a 12 meses por lo que no fue posible establecer estas comparaciones entre grupos de edad.

En este estudio tampoco se encontró relación de la frecuencia del cepillado dental con la frecuencia de caries. Otros estudios han encontrado una asociación entre puntajes de ceo-d más bajos con el inicio del cepillado dental más temprano en la vida y con mayor frecuencia de cepillado por día. ${ }^{29}$ La discrepancia de nuestros hallazgos podría relacionarse con la eficacia del cepillado, que, aunque pueda ser frecuente no logra eliminar la placa dentobacteriana eficientemente.

Respecto a la presencia de placa dentobacteriana y su relación con la frecuencia de caries, nuestros hallazgos son similares a lo encontrado en otros estudios. $^{27-30}$ La evidencia sugiere que la composición bioquímica y micro bacteriana de la placa y las alteraciones que esta sufre derivadas del consumo de azúcar están relacionadas a la caries. ${ }^{31}$ En relación a la posible asociación entre la salud dental de la madre y la de su hijo, existe evidencia contradictoria. ${ }^{32-33}$

Algunos trabajos sugieren que la madre es una fuente importante de transmisión de la infección en los niños menores de dos años debido al contacto íntimo con sus hijos. Por ejemplo, un trabajo en el que se estudiaron 30 parejas de madrehijo encontraron que la caries materna no tratada casi duplicó las probabilidades de caries nuevas en su hijo hasta por 3 superficies (referencia). Dela misma forma, se han encontrado paralelos entre hábitos de higiene, frecuencia y tipo de alimentos ingeridos entre la madre y sus hijos. ${ }^{33}$ Por otro lado, y al igual que lo encontrado en este estudio, otros trabajos no han registrado relación entre estas variables y han atribuido la presencia de caries a otros factores ajenos al comportamiento de la madre. ${ }^{32-34}$ 


\section{Conclusiones}

El presente estudio encontró una prevalencia de CIT-S del $81.8 \%$ entre preescolares evaluados. El aumento de la edad y la presencia de placa dentobacteriana en niveles deficientes son factores asociados a la frecuencia de caries. El carácter reversible de $2 / 3$ de las lesiones encontradas demanda intervenciones preventivas de salud dental en esta población.

\section{Referencias bibliográficas}

1. Fejerskov O. Changing paradigms in concepts on dental caries: Consequences for oral health care. Caries Research [en linea]. 2004;38(3):182-91. [consultado 12 Ago 2019] Disponible en: https://www.researchgate. net/publication/8552028_Changing_Paradigms_in_Concepts_on_Dental_Caries_Consequences_for_ Oral_Health_Care.

2. Acharya $S$, Tandon $S$. The effect of early childhood caries on the quality of life of children and their parents. Contemp Clin Dent [en linea]. 2011;2(2):98-101. [consultado 5 Oct 2018] Disponible en: https:// www.ncbi.nlm.nih.gov/pmc/articles/PMC3180838/.

3. Sheiham A, James WPT. A reappraisal of the quantitative relationship between sugar intake and dental caries: The need for new criteria for developing goals for sugar intake. BMC Public Health [en linea]. 2014; 14: 863. [consultado 29 Oct 2018] Disponible en: https://bmcpublichealth.biomedcentral.com/ articles/10.1186/1471-2458-14-863.

4. Zafar S, Yasin S, Siddiqi A. Early childhood caries: etiology, clinical considerations, consequences and management. International Dentistry Sa [en linea]. 2009;11(4):24-36. [consultado 4 Abr 2007] Disponible en: http://www.moderndentistrymedia.com/July_aug2009/zafar.pdf.

5. America's Pediatric Dentistry. State of little teeth report: an examination of the epidemic of tooth decay among our youngest children [en linea]. Chicago: AAPD; 2014 [consultado 17 Mayo 2017]. Disponible en: https://www.aapd.org/globalassets/assets/1/7/state_of_little_teeth_final.pdf

6. Arora A, Schwarz E, Blinkhorn AS. Risk factors for early childhood caries in disadvantaged populations. J Investig Clin Dent [en linea]. 2011;2(4):223-8. [consultado 13 Jun 2018] Disponible en: https:// onlinelibrary.wiley.com/doi/abs/10.1111/j.2041-1626.2011.00070.x.

7. Vargas CM, Ronzio CR. Disparities in early childhood caries. BMC Oral Health [en linea]. 2006;6 Suppl 1:S3. [consultado 12 Nov 2017] Disponible en: https://bmcoralhealth.biomedcentral.com/articles/10.1186/14726831-6-S1-S3.

8. Gimenez T, Bispo BA, Souza DP, Vigano ME, Wanderley MT, Mendes FM, et al. Does the Decline in Caries Prevalence of Latin American and Caribbean Children Continue in the New Century? Evidence from Systematic Review with Meta-Analysis. PLoS One [en linea]. 2016;11(10):e0164903. [consultado 7 Jul 2018] Disponible en: https://www.ncbi.nlm.nih.gov/pmc/articles/PMC5074528/.

9. Campos B. Nivel de riesgo de desarrollar caries dental en infantes de 0 a 3 años de edad [tesis Salud Publica en línea]. Guatemala: Universidad San Carlos de Guatemala, Facultad de Medicina, Escuela de Posgrado Salud Publica; 2015. [consultado 9 Sept 2018]. Disponible en: http://www.repositorio.usac.edu. gt/1904/1/Brenda\%20Carolina\%20Campos\%20Rodr\%C3\%ADguez.pdf

10. Sheiham A. Dental caries affects body weight, growth and quality of life in pre-school children. $\mathrm{Br}$ Dent J [en linea]. 2006;201(10):625-6. [consultado 16 Abr 2018] Disponible en: https://www.nature.com/ articles/4814259.

11. Newman MG, Takei HH, Carranza FA. Carranza's clinical periodontology. 9th ed. Philadelphia: W.B. Saunders Co; 2002.

12. Oral health surveys: Basic methods. $5^{\mathrm{a}}$ ed. Geneva: World Health Organization; 2013.

13. Joseph C, Bourgeois D, Muller Bolla M. DMF from WHO basic methods to ICDAS II advanced methods: a systematic review of literature. Odonto-stomatologie tropicale = Tropical dental journal. [en linea]. 2012; 35: 5-11. [consultado 12 Mar 2018] Disponible en: https://www.ncbi.nlm.nih.gov/pubmed/23316595

14. Iranzo Cortés J, Montiel-Company J, Almerich-Silla J. Caries diagnosis: agreement between WHO and ICDAS II criteria in epidemiological surveys. Community Dental Health [en linea]. 2013; 30:108-11. [consultado 11 Ene 2019] Disponible en: https://www.ncbi.nlm.nih.gov/pubmed/23888541 
15. Viana SVC, Piovesan C, Imparato Jcp, Raggio Dp, Almeida Ars, Bonini GC. Prevalence of dental caries in preschool children by ICDAS diagnostic methodology. Pesqui. bras. odontopediatria clín. integr. [en línea]. 2015; 15(1):291-300. [consultado 2018 Feb 22] Disponible en: http://revista.uepb.edu.br/index. $\mathrm{php} / \mathrm{pboci} /$ article/view/2914/pdf

16. Ismail AI, Sohn W, Lim S, Willem JM. Predictors of dental caries progression in primary teeth. J Dent Res [en linea]. 2009;88(3):270-5. [consultado 1 Jul 2018] Disponible en: https://pdfs.semanticscholar.org/5a3f/ a2eb1266b557f34665aaab9782aaa31153bd.pdf.

17. Ueda EMO, Dezan CC, Frossard WTG, Salomão F, Morita MC. Prevalence of dental caries in 3- and 5-yearold children living in a small Brazilian City. J. Appl. Oral Sci [en linea]. 2004;12(1):34-8. [consultado 12 Ene 2018] Disponible en: http://www.scielo.br/scielo.php?script=sci_arttext\&pid=S1678-77572004000100007.

18. Šačić L, Marković N, Arslanagić Muratbegović A, Zukanović A, Kobašlija S. The prevalence and severity of early childhood caries in preschool children in the Federation of Bosnia and Herzegovina. Acta Med Acad [en linea]. 2016;45(1):19-25. [consultado 7 Ago 2019] Disponible en: http://ama.ba/index.php/ama/ article/view/267/pdf.

19. Shaghaghian S, Abolvardi M, Akhlaghian M. Factors Affecting dental caries of preschool children in Shiraz. J Dent [en linea]. 2018;19(2):100-8. [consultado 13 Sep 2019] Disponible en: https://www.ncbi. nlm.nih.gov/pmc/articles/PMC5960729/

20. Stephen A, Krishnan R, Ramesh M, Kumar VS. Prevalence of early childhood caries and its risk factors in 18-72 month old children in Salem, Tamil Nadu. J Int Soc Prev Community Dent. [en linea]. 2015;5(2):95102. [consultado 10 Sep 2015] Disponible en: https://www.ncbi.nlm.nih.gov/pmc/articles/PMC4415336/

21. Hultquist AI, Bågesund M. Dentin caries risk indicators in 1-year-olds. A two year follow-up study. Acta Odontol Scand [en linea]. 2016;74(8):613-9. [consultado 13 Sep 2019] Disponible en: https://www. tandfonline.com/doi/full/10.1080/00016357.2016.1227085

22. Deeley K, Letra A, Rose EK, Brandon CA, Resick JM, Marazita ML, et al. Possible association of amelogenin to high caries experience in a Guatemalan-Mayan population. Caries Research [en linea]. 2008;42(1):8-13. [consultado 14 Mayo 2018] Disponible en: https://www.karger.com/Article/Abstract/111744.

23. Kato H, Tanaka K, Shimizu K, Nagata C, Furukawa S, Arakawa M, et al. Parental occupations, educational levels, and income and prevalence of dental caries in 3-year-old Japanese children. Environ Health Prev Med [en linea]. 2017;22(1):80. [consultado 22 Jul 2018] Disponible en: https:/environhealthprevmed. biomedcentral.com/articles/10.1186/s12199-017-0688-6.

24. Flores M, Montenegro B. Relación entre la frecuencia diaria de consumo de azúcares extrínsecos y la prevalencia de caries dental. Revista Estomatológica Herediana [en linea]. 2005;15(1):36-9. [consultado 28 Mayo 2018] Disponible en: http://www.redalyc.org/pdf/4215/421539343007.pdf.

25. Head D, A Devine D, Marsh PD. In silico modelling to differentiate the contribution of sugar frequency versus total amount in driving biofilm dysbiosis in dental caries. Sci Rep [en linea]. 2017;7(1):17413. [consultado 17 Oct 2018] Disponible en: https://www.nature.com/articles/s41598-017-17660-z.

26. Harris R, Nicoll AD, Adair PM, Pine CM. Risk factors for dental caries in young children: A systematic review of the literature. Community Dental Health [en linea]. 2004;21 Suppl 1:71-85. [consultado 29 Oct 2018] Disponible en: https://www.researchgate.net/publication/8630271_Risk_factors_for_dental_caries_ in_young_children_A_systematic_review_of_the_literature.

27. Victora CG, Bahl R, Barros AJD, França GVA, Horton S, Krasevec J, et al. Breastfeeding in the 21st century: Epidemiology, mechanisms, and lifelong effect. The Lancet [en linea]. 2016;387(10017):475-90. [consultado 17 Oct 2017] Disponible en: https://www.thelancet.com/journals/lancet/article/PIIS01406736(15)01024-7/fulltext.

28. Peres KG, Nascimento GG, Peres MA, Mittinty MN, Demarco FF, Santos IS, et al. Impact of Prolonged Breastfeeding on Dental Caries: A Population-Based Birth Cohort Study. Pediatrics. [en linea]. 2017;140(1). [consultado 18 Mayo 2018] Disponible en: https://pediatrics.aappublications.org/content/140/1/ e20162943.long

29. Elidrissi SM, Naidoo S. Prevalence of dental caries and toothbrushing habits among preschool children in Khartoum State, Sudan. Int Dent J [en linea]. 2016;66(4):215-20. [consultado 14 de Oct 2018] Disponible en: https://onlinelibrary.wiley.com/doi/abs/10.1111/idj.12223.

30. Mohebbi SZ, Virtanen JI, Vahid-Golpayegani M, Vehkalahti MM. Early childhood caries and dental plaque among 1-3-year-olds in Tehran, Iran. J Indian Soc Pedod Prev Dent [en linea]. 2006;24(4):177-81. [consultado 8 Mayo 2017] Disponible en: https://www.researchgate.net/publication/6617601_Early_ childhood_caries_and_dental_plaque_among_1-3-year-olds_in_Tehran_Iran.

31. Hemadi AS, Huang R, Zhou Y, Zou J. Salivary proteins and microbiota as biomarkers for early childhood caries risk assessment. Int J Oral Sci [en linea]. 2017 ;9(11):e1. [consultado 14 Oct 2018] Disponible en: http://europepmc.org/articles/pmc5775330. 
32. Hirooka LB, Mestriner-Junior W, Mestriner SF, Nunes SAC, Lemos PN, Franco LJ. Dental caries in mother-child pairs from Xingu. Braz. J. Oral Sci [en linea]. 2014 ;13(1):43-6. [consultado 29 Oct 2017] Disponible en: http://www.scielo.br/scielo.php?script=sci_arttext\&pid=S1677-32252014000100043.

33. Mannaa A, Carlén A, Lingström P. Dental caries and associated factors in mothers and their preschool and school children-A cross-sectional study. Journal of Dental Sciences [en linea]. 2013;8(2):101-8. [consultado 29 Oct 2018] Disponible en: https://www.researchgate.net/publication/259169354_Dental_ caries_and_associated_factors_in_mothers_and_their_preschool_and_school_children-A_crosssectional_study.

34. Weintraub JA, Prakash P, Shain SG, Laccabue M, Gansky SA. Mothers' caries increases odds of children's caries. J Dent Res [en linea]. 2010;89(9):954-8. [consultado 6 Mar 2017] Disponible en: https://www. semanticscholar.org/paper/Mothers'-caries-increases-odds-of-children's-Weintraub-Prakash/5862724a4 517a9e85da75cb1e1b64ce3ae55a71d

Recibido: 07/02/2020

Aceptado: 08/04/2020

Correspondencia: Barbara Bustamante correo: barbara.bustamante.castillo@gmail.com 